January 1992

\title{
Delusional Misidentification Syndromes
}

\author{
Zeljko Jocic, M.D. \\ University of North Dakota, Fargo, North Dakota
}

Follow this and additional works at: https://jdc.jefferson.edu/jeffjpsychiatry

Part of the Psychiatry Commons

Let us know how access to this document benefits you

\section{Recommended Citation}

Jocic, M.D., Zeljko (1992) "Delusional Misidentification Syndromes," Jefferson Journal of Psychiatry. Vol. 10 : Iss. 1 , Article 4.

DOI: https://doi.org/10.29046/JJP.010.1.001

Available at: https://jdc.jefferson.edu/jeffjpsychiatry/vol10/iss1/4

This Article is brought to you for free and open access by the Jefferson Digital Commons. The Jefferson Digital Commons is a service of Thomas Jefferson University's Center for Teaching and Learning (CTL). The Commons is a showcase for Jefferson books and journals, peer-reviewed scholarly publications, unique historical collections from the University archives, and teaching tools. The Jefferson Digital Commons allows researchers and interested readers anywhere in the world to learn about and keep up to date with Jefferson scholarship. This article has been accepted for inclusion in Jefferson Journal of Psychiatry by an authorized administrator of the Jefferson Digital Commons. For more information, please contact: JeffersonDigitalCommons@jefferson.edu. 


\title{
Delusional Misidentification Syndromes
}

\author{
Zeljko Jocic, M.D.
}

\begin{abstract}
Delusional misidentification syndromes are reviewed by their phenomenology, epidemiology, clinical characteristics, associated clinical findings, etiological theories, diagnostic evaluation, and treatment. Related neuropsychiatric syndromes are described and distinctions between them and delusional misidentification syndromes addressed. Current psychological and biologic-cognitive theories are briefly discussed. These peculiar phenomena are probably more frequent than previously thought, if they are specifically sought and recognized. Organic factors play a definitive role in their occurrence and two brain regions, frontal and temporal, are presumably dysfunctional.
\end{abstract}

\section{INTRODUCTION}

Delusional misidentification syndromes are a group of delusional phenomena in which patients misidentify familiar person, objects, or self, and believe that they have been replaced or transformed. These syndromes are delusional because the misidentifications are false and are not correctable by experience or reason. So far, many different sub-types have been identified, but most authors categorize them into four main syndromes (see table 1). Most reports in the literature are related to the Capgras syndrome which is the best-known. In many cases, two or more misidentification syndromes and related phenomena are present in the same patient $(5,6)$, which may indicate similar underlying pathophysiological mechanisms.

\section{EPIDEMIOLOGY}

Delusional misidentification syndromes seldom appear independent of comorbid pathology. They have been reported in association with other psychiatric disorders in $60 \%$ to $75 \%$ of cases and organic illnesses in $25 \%$ to $40 \%$ of cases. The most common psychiatric diagnoses have been: paranoid schizophrenia, schizoaffective psychosis, and bipolar disorder. In the last 20 years, reports have increasingly stressed the etiologic importance of a variety of conditions that have been found in the patients with misidentification syndromes, including: cerebrovascular disease, post-traumatic encephalopathy, temporal lobe epilepsy, post-encephalitic parkinsonism, viral encephalitis, migraine, vitamin B12 deficiency, hepatic encephalopathy, hypothyroidism, pseudoparathyroidism, and dementia $(7,8)$. It seems possible that these syndromes are generally organic and acquired and that their earlier association with the heritable major psychotic disorders may reflect failure to identify organic illness. 


\section{TABLE 1.}

Capgras Syndrome

Fregoli Syndrome

Syndrome of Intermetamorphosis

Syndrome of Subjective Doubles
Belief that a familiar person or object has been replaced by a nearly identical duplicate or impostor.

Belief that a familiar person acquires different physical identities while the psychological identity remains the same, i.e., the familiar person disguises himself as others.

Belief that another person has changed both his physical and psychological identities, i.e., has been transformed into another.

Belief that another person has been physically transformed into the patient's own self.

Misidentification syndromes are more frequent in females (75\%). Age of onset varies from 12 to 78, with an average in the early 40's (9). In more than 4/5 of patients, the onset is after the age of 30 (10). Family history of psychosis is reportedly present in $50 \%$ of patients (11), which calls into question the emphasis upon acquired organic origins of these syndromes, although genetic vulnerability for organicallyinduced misidentification psychosis might be an important etiologic factor. The prevalence of these syndromes is still a controversial issue. Traditionally, they have been viewed as rare. Christodoulou, et al. (12), needed 6 years to assemble 11 patients. Kiriakos and Ananth (13) found thirteen cases in eight years. Recently, in more systematic studies $(8,14-16)$ in different settings, it has been reported that the incidence and prevalence of misidentification syndromes are higher than previously thought, especially among certain patient groups. Dohn and Crews (8), for example, collected 25 patients in 13 months, the largest population of patients with Capgras syndrome studied at one time. These authors found an incidence of $5.3 \%$ of all psychiatric admissions at their university hospital. Prevalence among patients identified as schizophrenic was $15 \%$. They postulated an estimated prevalence of $0.12 \%$ in the general population for Capgras syndrome and proposed that misidentification syndromes would be found not to be rare if they were specifically sought and recognized.

\section{CLINICAL CHARACTERISTICS}

The clinical presentation is markedly paranoid, with intense suspiciousness. Depersonalization/derealization frequently precede the onset of misidentification. The mode of onset is either abrupt, closely following the course of an acute psychotic or medical episode, or less frequently, progressive (17). In the case of Capgras syndrome, the most frequent doubles are the spouse (if the patients were married, divorced, or separated) and siblings (if the patients were single) (8). The emotional relationship of the patient to the object of misidentification is not necessarily negatively charged (18). The important aspect of the relationship appears to be its intensity, rather than its positive or negative characteristics (12). Hostility is seen much more frequently in those patients diagnosed as schizophrenic than in other 
patients (19). Other delusions, in addition to the delusion of misidentification, are present in the majority of cases (11). At the onset of misidentification, many patients were perplexed and carefully examined the physical characteristics and behavior of those persons whose identity they questioned. Subsequently, they gained "psychotic insight" and they became convinced of the existence of "doubles" or "impostors," with no further need for detailed examination (18). The double is usually assumed to have evil intent. During the course of illness the number of "doubles" may expand, often involving medical personnel. The prognosis of the misidentification syndromes appears to depend upon the reversibility of the underlying organic or psychotic condition. Occasionally they are persistent or chronic with a degree of "autonomy" (18). During relapse, which follows remission, most patients experience return of the same misidentification syndrome that they had had during the original acute illness. Patients diagnosed as schizophrenic with Capgras syndrome are reported to show less deterioration over time than other schizophrenic patients, which could be explained by: (1) the observation that misidentification syndromes are usually present in paranoid schizophrenia, which is believed to produce less severe personality disintegration; or (2) the possibility that these patients have been misdiagnosed as schizophrenic, which is suggested by resemblance of their EEG changes to those of the psychosis of epilepsy, in which deterioration is less common and severe (17).

\section{RELATED NEUROPSYCHIATRIC SYNDROMES}

Reduplicative paramnesia, a term used for the first time by Pick (20), or reduplication (21) is a belief that place, person, time, events, self, and body parts have been duplicated or relocated. It usually occurs in the situation of acute cerebral lesions with initial confusional or amnestic states. Some authors $(22,23)$ believe that reduplicative paramnesia, Capgras syndrome, and other misidentification syndromes are very similar or the same phenomena, with the only differences being in the sphere of misidentification (24). Signer (25), on the other hand, recognizes that similarities exist but believes that the differences are important; in the reduplicative paramnesia there is no naming of the duplicate as an impostor and there is the presence of double orientation (simultaneous and separate existence of the original and the duplicate).

Autoscopy is the hallucinatory or pseudo-hallucinatory experience of seeing one's body at a distance. Patients with autoscopy accept a double as their "real self," with the feeling of belonging (26); they often have partial insight (9). In misidentification syndromes the double is psychologically different and is not perceived, but is believed in (delusion rather than perceptual disturbance). Autoscopy resembles the syndrome of subjective doubles, and some authors believe that autoscopy provides background on which misidentification syndromes, such as the syndrome of subjective doubles, may develop by the process of delusional elaboration of hallucinatory experience (8).

Prosopagnosia is the non-recognition of familiar faces and other familiar objects. It occurs in the presence of bilateral lesions in the mesial occipitotemporal region (27). Two reports in the literature $(28,29)$ have suggested that prosopagnosia may be 
the organic substrate for Capgras syndrome, but patients with prosopagnosia fail totally to recognize previously known faces and non-visual cues trigger immediate recognition of the identity of familiar persons, which is not the case in the true misidentification syndromes (9).

Jamais $v u$ is the inappropriate absence of familiarity in seeing situations and places (30). It is closely related to the phenomenon of derealization. Usually, its duration is brief, measured in seconds or minutes, and reality testing is preserved providing an intrusion rather than delusion.

\section{CLINICAL FINDINGS}

It has been reported that misidentification syndromes have very high incidence of EEG changes, ranging from mild and diffuse abnormalities to "epileptic" discharges. The most common findings are bilateral paroxysmal slow waves (24). Somatosensory evoked potential study in two cases has shown asymmetry of the late component in the right hemisphere, indicating disturbance in the analysis of information in the association areas of the brain (31). CT findings have shown bilateral frontal and temporal lobe atrophy $(32,33)$, right hemispheric lesions superimposed either on diffuse cortical atrophy (5) or on bifrontal lesions (22), or only right temporo-parieto-occipital lesion (23). One report of MRI scan (34) revealed bilateral subcortical lesions in the occipitotemporal and frontal regions. Neuropsychological testing has shown a pattern of deficits indicating fronto-temporal (35) or diffuse cortical dysfunction (24) as the most prevalent findings. MMPI evaluations reveal significant depression, paranoia, isolation and constricted expression of emotions (36). Rorschach testing shows unusual experiences of the environment (37); responses are determined by small details of the pictures (piecemeal-style perception), which suggests the presence of right hemispheric and frontal lobe dysfunction (38).

\section{ETIOLOGICAL THEORIES}

\section{Psychological theories}

Early explanations of these syndromes are based on psychodynamic concepts and principles, such as Oedipus complex, defence mechanisms, splitting, regression, etc.

Ambivalence theory proposes that ambivalent feelings toward the person who is believed to have been replaced by an impostor lead to psychotic solution through denial and displacement. The double can be treated with hostility without guilt, especially because of the projection of bad attitude onto the double (39). At the same time, positive feelings are expressed for the imaginary original, who is idealized.

Depersonalization/derealization theory is based on the presumption that these patients perceive environment and their body in an unusual way which is experienced far more strongly in relation to persons or objects with whom patients have strong affective ties. This sense of strangeness has been projected to others and delusionally elaborated as "projected depersonalization" (40). Christodoulou (41) has found 
symptoms of depersonalization/derealization in more than a half of his patients especially just before or at the onset of delusions. For him, derealization is a projected form of depersonalization, and when it is channeled in a person(s) or objects then a delusional misidentification can result.

Regression theory (42) states that a compromise of higher cerebral functioning results in a reactivation of primitive modes of thinking which are characterized by the theme of doubles and dualisms, also found in myths, primitive religion, and literature. Other variants of this theory propose that deep regression reactivates a developmental stage prior to the establishment of object constancy, where there is splitting of objects into all good or all bad and absence of self-object differentiation (Kleinien paranoid-schizoid position).

\section{Biological or Cognitive Theories}

In the last 20 years, delusional misidentification syndromes have been reported in a variety of organic illnesses and conditions. Many case reports and several studies on series of patients have been able to identify focal and diffuse abnormalities of the central nervous system associated with these syndromes. That created a need for an etiopathological explanation taking into account these findings and offering an hypothesis about a possible neural substrate for delusional misidentification syndromes. It has been noted that if only one brain hemisphere is dysfunctional, typically that has been the right hemisphere. Since it is involved in the analysis of visuospacial tasks, disturbances in the right hemisphere could affect visuospacial perception and analysis and cause inability to correctly integrate perception with memories of familiar persons or objects (43). Support for this hypothesis comes from the finding that late component of somatosensory evoked responses is asymmetrical on the right side (31) and that neuropsychological testing sometimes shows selective disturbance of visuospacial function (44).

Staton et al. (23) suggest another explanation. They propose that delusional misidentification is the consequence of anatomical-functional disconnection in the deep right temporo-parieto-occipital region which causes deficit in the memory integration. New memory registration is disconnected from past memory stores and orientation to the present is based solely on recollections from the past causing the phenomenon of duplication of person(s) or objects. If frontal lobe dysfunction is present, that might facilitate a continuing misinterpretation of reality.

Joseph's theory is based on interhemispheric disconnection (24), in which each hemisphere decodes sensory information partially, so that integrative "image" is possible only if information flow between the hemispheres is not compromised. In the situation of interhemispheric disconnection each hemispheric "image" is formed simultaneously causing two separate perceptions of person, object, place, or time.

Van Lancker offers another perspective (45). She explains delusion of misidentification as a disturbance in experience of familiarity (personal relevance). Feelings of familiarity include affective interaction between subject and object. Various categories of stimuli, which are idiosyncratic and personal, can receive the "familiarity" 
attribute: proper names, faces, voices, persons, phrases, objects, or topography. In the situation of the right hemisphere dysfunction when there is a defective perception and processing of affective information, that could lead to simultaneous intellectual recognition and affective non-recognition (feeling of unfamiliarity) of known person(s) and/or objects (43).

\section{DIAGNOSTIC EVALUATION}

The misidentification syndromes are seldom discrete disorders. They usually occur in association with other psychiatric or organic conditions. It is therefore necessary to perform a comprehensive evaluation in order to rule in or out other comorbid conditions. Proper evaluation should include: (1) psychiatric history and mental status examination, using past history, family history, and clinical clues for distinguishing psychiatric disorders from organic mental syndromes; (2) physical examination (including detailed neurological examination); (3) laboratory tests (CBC, BUN, Cr, electrolytes, blood glucose, thyroid function tests, liver function tests); (4) chest x-rays; (5) EEG; (6) CT scan of the head, if indicated; and (7) neuropsychological testing, if indicated.

\section{TREATMENT}

Treatment is dictated by the nature of the underlying organic or psychiatric conditions. Improvements in an organic illness may be accompanied by gradual remission of delusional ideation. If a primary psychotic illness appears to be present, antipsychotic medications with low anticholinergic potencies are recommended, since atropinic toxicity has been implicated as a causative factor (46). Lithium, carbamazepine, or valproic acid, with or without antipsychotic medication, could be used in the case of bipolar or schizoaffective psychoses. If EEG changes are prominent, carbamazepine, or valproic acid might be preferable choices.

\section{DISCUSSION}

Almost 90 years have passed since the first report on misidentification syndromes appeared in the medical literature (20). They are still puzzling, psychopathological phenomena. Although recent epidemiological studies give the impression that delusional misidentification syndromes are encountered more frequently than was previously thought, we remain unsure of the precise prevalence. In future prospective studies, use of a specially designed questionnaire (47) might give more accurate information about incidence and prevalence. It is evident that patients should be asked about their delusions in a systematic way, since they are unlikely to spontaneously divulge convictions of duplication. Delusional misidentification syndromes have been found in many different medical and psychiatric conditions. It would be important to know if there is a common neuropathologic disturbance in all of them. The recent literature describing organic findings is very suggestive, especially the 
very high incidence of EEG changes. More extensive electrophysiologic examinations should be conducted, using Brain Electrical Activity Mapping, cognitive evoked potentials, magnetoencephalography, sleep-derived EEG with nasopharyngeal leads, with particular reference to temporolimbic functioning, since electrical stimulation and epileptic discharge in that area have elucidated similar phenomena (48-51). One might hypothesize that premorbid suspiciousness coupled with temporolimbic dysfunction could predispose an individual to experiences illusions of (un)familiarity. If frontal lobe dysfunction existed at the same time, insight and judgement might be sufficiently impaired to permit development of delusions of (un)familiarity. Controlled CT scan studies (33) revealing bilateral frontotemporal atrophy in the patients with delusional misidentification syndromes is compatible with this explanation.

\section{REFERENCES}

1. Capgras, J, and Reboul-Lachaux, J: L'illusion des sosies dans un delire systematise chronique. Bull Soc Clin Med Ment; 11:6-16, 1923

2. Courbon, P, and Fail, G: Syndrome d'illusion de Fregoli et schizophrenie. Bull Soc Clin Med Ment; 15:121-124, 1927

3. Courbon, P, and Tusques, J: Illusion d'intermetamprphose et de charme. Annls medpsychol; 90:401-405, 1932

4. Christodoulou, GN: Syndrome of subjective doubles. Am J Psychiatry; 135:249-251, 1978

5. Joseph, AB: Bitemporal atrophy in a patient with Fregoli syndrome, syndrome of intermetamorphosis, and reduplicative paramnesia (letter). Am J Psychiatry; 142:146-147, 1985

6. Atwal, $\mathrm{S}$, and $\mathrm{Khan}, \mathrm{MH}$ : Coexistence of Capgras and its related syndromes in a single patient. Aust N Z J Psychiatry; 20:496-498, 1986

7. Cummings, JL: Clinical Neuropsychiatry; Grune and Stratton, Inc., 1985

8. Dohn, HH, and Crews, EL: Capgras syndrome: A literature review and cases series, Hillside J Clin Psychiatry; 8:56-74, 1986

9. Berson, RJ: Capgras' syndrome, Am J Psychiatry; 140:969-978, 1983

10. Enoch, MD: Whose Double?, in The Delusional Misidentification Syndromes, by G.N. Christodoulou (editor), Biblioteca Psychiatrica; No 164, Karger, 1986

11. Kimura, S: Review of 106 cases with the syndrome of Capgras, in The Delusional Misidentification Syndromes, by GN Christodoulou (editor), Biblioteca Psychiatrica; No 164, Karger, 1986

12. Christodoulou, GN: The Syndrome of Capgras, Br J Psychiatry; 130:556-564, 1977

13. Kiriakos, R, and Ananth, J: Review of 13 cases of Capgras syndrome, Am J Psychiatry; 137:1605-1607, 1980

14. Walter-Ryan, WG: Capgras syndrome and misidentification (letter to the editor), Am J Psychiatry; 143:126, 1986

15. Fishbain, DA: The frequency of Capgras delusions in a psychiatric emergency service, Psychopathology; 20:42-47, 1987

16. Morrison, JR: Capgras delusion in a private practice, J Clin Psychiatry; 41:355-356, 1980

17. Christodoulou, GN: Course and outcome of the delusional misidentification syndromes, in The Delusional Misidentification Syndromes, by GN Chrostodoulou (editor), Biblioteca Psychiatrica; No 164, Karger, 1986 
18. Christodoulou, GN: Course and prognosis of the syndrome of doubles, J Nerv Ment Dis; 166:68-72, 1978

19. Wallis, G: Nature of the misidentified in the Capgras syndrome, in The Delusional Misidentification Syndromes, by GN Christodoulou (editor), Biblioteca Psychiatrica; No 164, Karger, 1986

20. Pick, A: Clinical Studies, III: On reduplicative paramnesia, Brain; 26:260-267, 1903

21. Weinstein, EA, and Burnham, DL: Reduplication and the syndrome of Capgras, Psychiatry; 54:78-88, 1991

22. Alexander, MP, Stuss, DT, and Benson, DF: Capgras syndrome: A reduplicative phenomenon, Neurology; 29:334-339, 1979

23. Staton, RD, Brumback, RA, and Wilson, H: Reduplicative paramnesia: A disconnection syndrome of memory, Cortex; 18:23-36, 1982

24. Joseph, AB: Focal central nervous system abnormalities in patients with misidentification syndromes, in The Delusional Misidentification Syndromes, by GN Chrisotdoulou (editor), Biblioteca Psychiatrica; No 164, Karger, 1986

25. Signer, SF: Capgras' Syndrome: The delusion of substitution, J Clin Psychiatry; 48:147150,1987

26. Damas Mora, JMR, Jenner, FA, and Eacott, SE: On hautoscopy or the phenomenon of the double: Case presentation and review of the literature, Br J Med Psychol; 53:75-83, 1980

27. Damasio, AR, Damasio, H, and Van Hoesen, GWQ: Prosopagnosia: Anatomic basis and behavioral mechanisms, Neurology; 32:331-341, 1982

28. Shraberg, D, and Weitzel, WD: Prosopagnosia and the Capgras syndrome, J Clin Psychiatry; 40:313-316, 1979

29. Hayman, MA, and Abrams, R: Capgras' syndrome and cerebral dysfunction, Br J Psychiatry; 130:68-71, 1977

30. Sno, HN, and Linszen, DH: The deja vu experience: Rememberance of things past?, Am J Psychiatry; 147:1587-1595, 1990

31. Wilcox, J, and Waziri, R: The Capgras syndrome and nondominant cerebral dysfunction, J Clin Psychiatry; 44:70-72, 1983

32. Joseph, $\mathrm{AB}$, and O'Leary, $\mathrm{DH}$ : Anterior cortical atrophy in Fregoly syndrome, J Clin Psychiatry; 48:409-411, 1987

33. Joseph, AB, O'Leary, DH, and Wheeler, HG: Bilateral atrophy of the frontal and temporal lobes in schizophrenic patients with Capgras syndrome: A Case-Control Study using CT, J Clin Psychiatry; 51:322-325, 1990

34. Lewis, SW: Brain imaging in a case of Capgras' syndrome, Br J Psychiatry; 150:117-121, 1987

35. Morrison, RL, and Tarter, RE: Neuropsychological findings related to Capgras syndrome, Biol Psychiatry; 19:1119-1128, 1984

36. MacCallum, WAG: The interplay of organic and psychological factors in the delusional misidentification syndromes, in The Delusional Misidentification Syndromes, by GN Christodoulou (editor), Biblioteca Psychiatrica; No 164, Karger, 1986

37. Stern, K, MacNaughton, D: Capgras syndrome, a peculiar illusionary phenomenon, considered with special reference to the Rorschach findings, Psychiatr Q; 19:139-163, 1945

38. Bidault, E, Luaute, JP, and Tzavaras, A: Prosopagnosia and the misidentification syndromes, in The Delusional Misidentification Syndromes, by GN Christodoulou (editor), Biblioteca Psychiatrica; No 164, Karger, 1986 
39. Sims, A: Psychopathology of schizophrenia with special reference to delusional misidentification, in The Delusional Misidentification Syndromes, by GN Christodoulou (editor), Biblioteca Psychiatrica; No 164, Karger, 1986

40. O'Reilly, R, and Malhotra, L: Capgras Syndrome-An unusual case and discussion of psychodynamic factors, Br J Psychiatry; 151:263-265, 1987

41. Christodoulou, GN: Role of depersonalization-derealization phenomena in the delusional misidentification syndromes, in The Delusional Misidentification Syndromes, by GN Christodoulou (editor), Biblioteca Psychiatrica; No 164, Karger, 1986

42. Sinkman, AM: The Capgras delusion: A critique of its psychodynamic theories, Am J Psychotherapy; 37:428-438, 1983

43. Wilcox, JA: The anatomical basis of misidentification, in The Delusional Misidentification Syndromes, by GN Chrisotdoulou (editor), Biblioteca Psychiatrica; No 164, Karger, 1986

44. Feinberg, TE, and Shapiro, RM: Misidentification-reduplication and the right hemisphere, neuropsichiatry, neuropsychology, and behavioral neurology; 2:39-48, 1989

45. Van Lancker, D: Personal relevance and the right hemisphere, brain and cognition, in press.

46. Waziri, R: The Capgras Phenomenon: Cerebral dysfunction with psychosis, neuropsychobiology; 4:353-359, 1978

47. Hakim, H, Verma, NP, and Greiffenstein, M: Pathogenesis of reduplicative paramnesia, J Neurol Neurosurg Psychiatry; 51:839-841, 1988

48. Gloor, P, Oliver, A, Quesney, LF, et al: The role of the limbic system in experiential phenomena of temporal lobe epilepsy, Ann Neurol; 12:129-144, 1982

49. Halgren, E, Walter, RD, Cherlow, DG, et al: Mental phenomena evoked by electrical stimulation of the human hippocampal formation and amygdala, Brain; 101:83-117, 1978

50. Ardila, A, Botero, M, Gomez, J, et al: Partial cognitive-dysmnestic seizures as a model for studying psychosis, Int J Neurosci; 38:11-20, 1988

51. Gloor, P: Experimental phenomena of temporal lobe epilepsy, Brain; 113:1673-1694, 1990 